\title{
First report of multiple drug-resistant gastrointestinal nematodes of sheep in Arbat District, Sulaymaniyah, Iraq detected by in vivo and in vitro methods
}

\author{
Hiewa Othman Dyary ${ }^{\boxplus}$, Hamasalih Qadir Banaz ${ }^{2}$ \\ ${ }^{1}$ Department of Basic Sciences, College of Veterinary Medicine, University of Sulaimani, Sulaymaniyah 46001, Iraq \\ ${ }^{2}$ Directorate of Veterinary Services in Sulaymaniyah, Sulaymaniyah 46001, Iraq \\ dyary.othman@univsul.edu.iq
}

Received: March 22, 2021

Accepted: August 3, 2021

\begin{abstract}
Introduction: The control of gastrointestinal nematodes (GINs) in small ruminants is principally dependent on anthelmintic therapy, which encounters the rising problem of anthelmintic resistance (AR) development. Veterinarians reported anthelmintic failure in several sheep farms in Arbat District, Sulaymaniyah, northern Iraq, which called for a systematic study about the efficacy of three commonly used drugs: albendazole, ivermectin, and levamisole. Material and Methods: A faecal egg count reduction test (FECRT) was conducted to elucidate the anthelmintics' efficacies, with coprocultures to determine the GINs parasitising sheep in the study area. Larval development assays (LDAs) were conducted to determine the drugs' median inhibitory concentrations $\left(\mathrm{IC}_{50}\right)$. Results: The FECRT revealed that AR was widespread to all three drugs, and ivermectin was the least effective, reducing the faecal egg counts (FECs) by $50.5 \%$ to $57.1 \%$. The coprocultures revealed that the GIN genera of sheep in Arbat District were Trichostrongylus, Nematodirus, and Trichuris, and the resistance was mainly due to Trichostrongylus species. The mean IC50 of albendazole, ivermectin, and levamisole were $0.073 \mathrm{ng} / \mathrm{mL}, 7.97 \mathrm{ng} / \mathrm{mL}$, and $1.43 \mathrm{ng} / \mathrm{mL}$, respectively. Conclusion: This study is the first Iraqi report of AR confirmed by both in vivo FECRT and in vitro LDA methods.
\end{abstract}

Keywords: albendazole, anthelmintic resistance, ivermectin, levamisole, Trichostrongylus, larval development assay.

\section{Introduction}

The Nematoda phylum includes many helminth species parasitising humans and domestic and wild animals (23). Gastrointestinal nematodes (GIN) are globally distributed in small ruminants and negatively impact animal health and production. All grazing sheep are exposed to GIN infections, which are regarded as one of the most important ovine diseases worldwide and cause weight loss, diarrhoea, and death (7). Production losses result from the loss of appetite, tissue and organ damage, reduced feed conversion, and deaths of heavily infected animals. The amounts of weight gain and milk production in infected animals are $15 \%$ and $22 \%$ less than those of noninfected animals, respectively, and in addition, the cost of anthelmintic therapies indirectly impacts livestock productivity (19).

Different strategies are implemented for GIN control in domestic animals, but anthelmintic therapy is the most widespread method of helminth control globally (9). The most commonly used anthelmintics are benzimidazoles, macrocyclic lactones, or imidazothiazoles (1). These are broad-spectrum drugs with wide safety margins, but their extensive use has led to anthelmintic resistance (AR) development, which occurs when a drug's recommended dosage cannot effectively treat the infected animal (8). The arising and spread of AR are accelerated by underdosing, repeated use of the same anthelmintic, and treatment of the whole flock (3).

Parasitic diseases and the emergence of AR are significant human health problems (25). This resistance is widespread in sheep-producing countries, is a persistent and growing issue in all parts of the world, and requires immediate attention (11). Several reports of AR development have been published in recent years, and it has become a global problem in sheep and goats in the last three decades $(2,22)$.

Successful helminth control strategies partially depend on accurately detecting AR in an area. However, several factors other than resistance may be responsible for anthelmintic therapy failure. For example, faulty 
flock drenching equipment may lead to livestock not being thoroughly dewormed. Anthelmintic underdosing due to inaccurate estimation of the animal's weight or anthelmintic dose is another common cause of anthelmintic failure (24).

Various in vitro and in vivo AR detection methods in GINs have been developed, but most of these tests are not adequately reliable, reproducible, or sensitive (24). The faecal egg count reduction test (FECRT) is the most commonly used method to estimate anthelmintic efficacy in vivo by comparing pre-treatment and posttreatment faecal egg counts (FECs) (4). Larval development assays (LDAs) are also considered reliable methods for investigating the progress of AR to different anthelmintic drugs, including albendazole, ivermectin, and levamisole (12).

One of the drawbacks of the FECRT is that it does not provide accurate information about the nematode species parasitising the tested animals and endowed with resistance. Some nematodes such as Teladorsagia colubriformis, Nematodirus spp., and Ostertagia circumcincta produce fewer eggs than other species such as Haemonchus contortus (24), and therefore species knowledge is important for sound interpretation of FECRT results. Hence, larval culture is used to determine the nematode species responsible for AR development.

In the Kurdistan Region, northern Iraq, the sheep population numbers more than three million head and is mainly raised on pasture grazing. Their farmers use anthelmintics to reduce the burden of GIN infection. The most commonly used dewormers are albendazole, ivermectin, and levamisole, administered via drenching. Recently, incidents of the common anthelmintics' failure were reported by veterinary practitioners on several farms in the Arbat District, one of the Sulaymaniyah districts in the Kurdistan Region of Iraq. Accordingly, this study investigated the occurrence of resistant sheep GINs on three farms in Arbat using in vivo FECRT, larval culture, and in vitro LDA.

\section{Material and Methods}

Farm selection and anthelmintic treatment. Anthelmintics are commonly used to control soiltransmitted nematodes in small ruminants in the north of Iraq. This study was conducted to investigate AR occurrence in Arbat District, northeastern Iraq, located $35.28^{\circ}-35.38^{\circ} \mathrm{N}$ and $45.13^{\circ}-45.29^{\circ} \mathrm{E}$. Veterinary practitioners have reported the failure of anthelmintic therapy on approximately ten sheep farms in the region.

Arbat District is located $23 \mathrm{~km}$ southeast of the Sulaymaniyah Governorate, Kurdistan Region, northern Iraq. The district comprises 71 villages, and mainly sheep and cattle farming represents the primary income source. The sheep population is around 65,000, and the animals are kept in different size flocks and raised on free pasture throughout the year.
Farmers in Arbat District drench their sheep with anthelmintics once or twice a year to control GIN infections. Avermectins are the most commonly used, followed by imidazothiazoles and benzimidazoles. Veterinary practitioners reported the therapeutic inefficiency of antinematodal drugs in several herds after drenching the sheep with several anthelmintics. These incidences urged the carrying out of a standardised study to investigate the efficacy of the commonly used anthelmintics in this region.

Ten farms were visited to investigate their management practices. A questionnaire was distributed among the farmers, putting several questions about the number, age, and sex of the sheep on each farm, the date of previous treatment, and the type of anthelmintic used. Farms were included in the study by meeting qualifying criteria: keeping $\geq 40$ sheep between the ages of six and eighteen months, grazing them on pasture, not having treated them with anthelmintics for the previous three months, and scoring a mean pre-treatment faecal egg count of $\geq 200$ eggs per gram (EPG) for their animals.

Three farms met the selection criteria and were included in this study. The combined number of sheep raised on the studied farms was 3,623, accounting for about $5.6 \%$ of Arbat's sheep population. The other farms were excluded for various reasons, such as carrying out treatment with anthelmintics in the previous 12 weeks, keeping too few animals of the required age, or the owners' declining to participate in the study.

Forty sheep of both sexes, 6 to 18 months old, were selected from each farm, divided into four groups of 10 , and tagged appropriately. The first group served as the control and was drenched with distilled water. Groups 2 to 4 were administered a single oral dose of albendazole, ivermectin, or levamisole, respectively.

The recommended anthelmintic dosages of $5.0 \mathrm{mg} / \mathrm{kg}$ of albendazole (Albenol-100), $0.2 \mathrm{mg} / \mathrm{kg}$ of ivermectin (Intermectin Drench), and $10.0 \mathrm{mg} / \mathrm{kg}$ of levamisole (Leva-200) were used. All drugs were produced by Interchemie (Venray, the Netherlands). Each animal's dose was calculated according to its weight and administered using an automatic drenching gun. The study protocol was submitted to and approved by the Animal Care and Use Committee (ACUC) at the College of Veterinary Medicine, University of Sulaimani.

Sample collection and faecal egg count reduction test. Faecal samples were collected twice from each sheep. The first sample was collected just before the anthelmintic administration, and the second was taken 14 days post-treatment. Faecal pellets (5-15 g) were taken directly from each animal's rectum, put in suitable containers, labelled, and stored in an icebox. The samples were brought to the Research Laboratory at the College of Veterinary Medicine, University of Sulaimani, and kept refrigerated overnight. An FEC was conducted for each sample applying a modified McMaster method. The procedure started with weighing $3.0 \mathrm{~g}$ of faeces into a container and adding $42.0 \mathrm{~mL}$ 
of water. The mixture was then homogenised using a laboratory stirrer and shaken until all pellets were broken up. The homogenised mixture was poured into a bowl through a $0.15 \mathrm{~mm}$ aperture, $20.0 \mathrm{~cm}$ diameter sieve. Fifteen millilitres of the filtrate were put into a centrifuge tube, and this was centrifuged for two minutes at $1,500 \mathrm{rpm}$. The supernatant was gently discarded, the tube was agitated to loosen the sediment, and a saturated sodium chloride solution was added to give the same volume $(15.0 \mathrm{~mL})$ as before. The tube was shaken by inverting five or six times, and immediately a sample was withdrawn with a pipette. The two chambers of a McMaster slide were filled with the withdrawn mixture. Nematode eggs were observed under an inverted microscope with $40 \times$ magnification power. All the nematode eggs under the two ruled grids (total volume of $0.3 \mathrm{~mL}$ ) were counted, and the final number was multiplied by 50 to give the EPG in the faecal sample (9).

The FECRT determines an anthelmintic's efficacy in vivo by comparing the post-treatment FEC to the pretreatment FEC and calculating the faecal egg count reduction (FECR). This was calculated as

$100 \times\left(1-\frac{\text { post-treatment } F E C \text { in the treatment group }}{\text { post-treatment } F E C \text { in the control group }}\right)$ and the $95 \%$ confidence interval as FECR $\pm 1.96 \sqrt{\text { variance }}$.

Faecal egg count reduction percentages below 95\% and/or lower $95 \%$ CI limits equal to or lower than $90 \%$ indicated resistance. If only one condition were met, resistance was suspected, and the herd was considered resistant if the FECR was $<95 \%$ and the lower CI was $<90 \%(4,10)$.

The efficacies of albendazole, ivermectin and levamisole in the study region were compared statistically using a one-way analysis of variance (ANOVA), followed by Duncan's post hoc test. The Statistical Package for the Social Sciences (SPSS version 24.0) program from IBM (Armonk, NY, USA) was used to conduct the statistical analysis.

Coprocultures. Faecal samples were collected before and after anthelmintic treatment, and $3.0 \mathrm{~g}$ of faecal pellets from each sheep on the same farm and in the same treatment group were mixed in a glass jar. Distilled water was added to the jar to bring the consistency to a suitable level, and the jar was incubated at $25^{\circ} \mathrm{C}$ for $7 \mathrm{~d}$ for third-stage nematode larval development. The pre-treatment samples were cultured to determine the GINs parasitising sheep in the study area. The post-treatment faecal samples were cultured to determine the genera that survived the anthelmintic therapy and acquired resistance.

After incubation for one week, the third-stage larvae (L3s) were collected by the larvoscopic Baermann technique, previously described by Coles et al. (4). The harvested larvae were put on a glass slide, stained with Lugol's iodine and covered with a coverslip. The L3s were examined under an inverted microscope $(100 \times$ magnification). The first 100 larvae detected in the slide were morphologically identified to the genus level (26), the proportion of each genus in the first
$100 \mathrm{~L} 3 \mathrm{~s}$ was calculated, and the reduction percentage of each nematode genus was determined using the following formula:

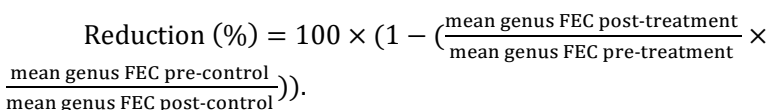

Larval development assay. Larval development assays (LDAs) were performed to assess the drugs' effect on the GIN eggs' hatchability and calculate their median inhibitory concentration $\left(\mathrm{IC}_{50}\right)$ in vitro. The samples used for the LDAs were from animals not treated with an anthelmintic for three months. The method described by Coles et al. (5) was implemented.

Tests were performed in 96-well microtitre plates. Nematode eggs were recovered from the faeces by passage through sieves with $250 \mu \mathrm{m}$ and $75 \mu \mathrm{m}$ aperture sizes, followed by centrifugation on a two-step sucrose gradient ( $10 \%$ and $25 \%$ sucrose). The eggs were recovered from the top layer of the $25 \%$ sucrose and washed over a $25 \mu \mathrm{m}$ sieve with water to remove residual sucrose.

The eggs were agitated gently in an $8.4 \mathrm{mg} / \mathrm{L}$ sodium hypochlorite solution for $12 \mathrm{~min}$ and subsequently washed with a large volume of water. The eggs had distilled water added in order that after the addition of amphotericin B (at a final concentration of $25.0 \mathrm{mg} / \mathrm{mL}$ ) and tylosin tartare (at a final concentration of $800 \mu \mathrm{g} / \mathrm{mL}$ ), $30 \mu \mathrm{L}$ contained 50-60 eggs, and the solution was used immediately for larval development assays (6). Assay plates were prepared by adding $150 \mu \mathrm{L}$ of nutrient broth to the wells of a 96-well plate. Egg solution in a $30 \mu \mathrm{L}$ volume was added to each well.

The microtitre plates were put in a humid chamber to prevent drying and incubated overnight at $26^{\circ} \mathrm{C}$. The next day, $2.0 \mu \mathrm{L}$ of a growth medium was added to each well (14). The medium constituents were Earle's salt solution $(10 \% \mathrm{v} / \mathrm{v})$, yeast extract $(1 \% \mathrm{w} / \mathrm{v})$, sodium bicarbonate $(1 \mathrm{mM})$, and saline solution $(0.9 \%$ sodium chloride $\mathrm{w} / \mathrm{v})$. The LDA used was a modified version of the method described initially by Taylor et al. (24). In the modified method, the culture medium comprised lyophilised Escherichia coli and $2.0 \mu \mathrm{L}$ of a solution of $E$. coli cells in lysogeny broth (LB) and $6.0 \mu \mathrm{L}$ of water. The final volume of the egg solution was adjusted to give 300 eggs $/ \mathrm{mL}$. Three concentrations of albendazole $(0.1 \mu \mathrm{g} / \mathrm{mL}, 0.3 \mu \mathrm{g} / \mathrm{mL}$, and $0.9 \mu \mathrm{g} / \mathrm{mL})$, levamisole $(1.0 \mu \mathrm{g} / \mathrm{mL}, 3.0 \mu \mathrm{g} / \mathrm{mL}$ and $9.0 \mu \mathrm{g} / \mathrm{mL})$ and ivermectin (3.5 ng/mL, $10.5 \mathrm{ng} / \mathrm{mL}$ and $31.5 \mathrm{ng} / \mathrm{mL}$ ) were tested.

The plates were incubated at $26^{\circ} \mathrm{C}$ for additional five days. After that, $10 \mu \mathrm{L}$ of Lugol's iodine solution was added to each well, and the content was put on a glass slide. The number of fully developed infectivestage larvae (L3) present in each well was counted. The percentage of L3 in the treatment wells was calculated by the formula $100 \times$ (number of L3 in T/number of L3 in $\mathrm{C}$ ), where $\mathrm{T}$ and $\mathrm{C}$ are the wells containing the test drugs and control wells, respectively. The $\mathrm{IC}_{50}$ of each anthelmintic was calculated using the Microsoft Excel 2016 program, and all tests were run in triplicate. 


\section{Results}

Determination of the tested drugs' efficacies in vivo. Gastrointestinal nematodes resistant to albendazole, ivermectin, and levamisole were present on the three farms. All the tested anthelmintics proved ineffective in reducing the post-treatment FEC by $95 \%$ (Fig. 1). However, the FECR by albendazole was significantly higher than those achieved by the other anthelmintics, and levamisole was more efficacious than ivermectin $(\mathrm{P}<0.05)$. Ivermectin reduced the FECR by $50.5 \%, 57.1 \%$, and $54.4 \%$ on the three farms.

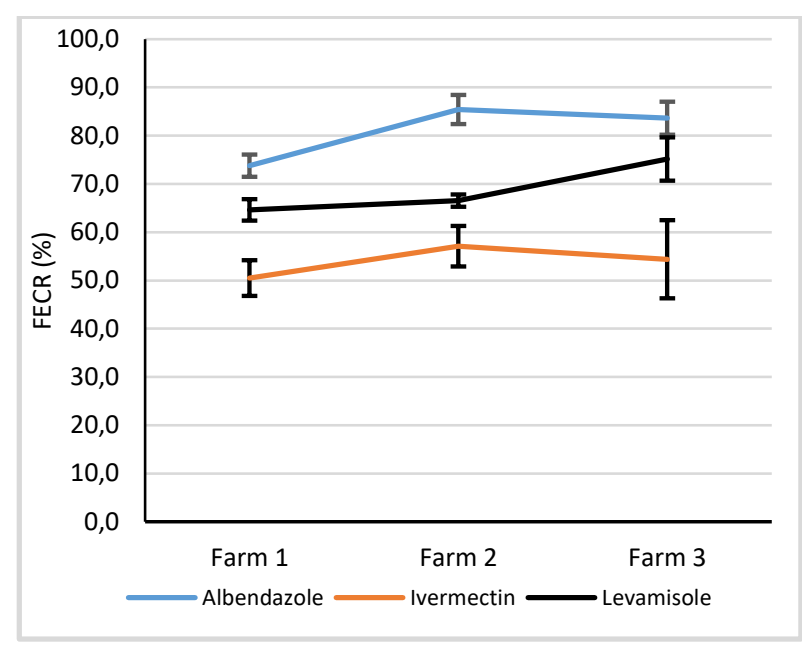

Fig. 1. Faecal egg count reduction (FECR) by albendazole, ivermectin, and levamisole on three farms in Arbat District, Sulaymaniyah, Iraq. All anthelmintics failed to reduce the post-treatment FEC by $95 \%$. Values represent the average FEC in 10 sheep (lines) and 95\% confidence intervals (error bars)

Gastrointestinal nematodes in the study area. The FEC in the pre-treatment samples ranged between 604.2 and 764.1 EPG. The larval culture revealed that Trichostrongylus spp. were the most common L3s (76\%-86\%), while Nematodirus spp. and Trichuris spp. were also detected (Table 1). Trichostrongylus species reduction did not reach $95 \%$ following treatment with any of the three drugs. The nematodes of this genus were reduced by $63.3 \%, 66.2 \%$, and $70.5 \%$ on the studied farms following levamisole treatment (Fig. 2). Trichostrongylus reduction ranged between $73.1 \%$ and $82.4 \%$ after albendazole treatment and between 49.3 and $52.4 \%$ after ivermectin treatment. These results indicated that the resistance is primarily caused by resistant Trichostrongylus species since they were the most prevalent in the pre-treatment samples.

Larval cultures of the post-treatment faecal samples revealed that Nematodirus was reduced by $50 \%$ in farm number 3 following albendazole treatment, indicating the emergence of albendazole-resistant Nematodirus. However, the genus was eliminated completely on the other farms.

The ivermectin-resistant GINs were Trichostrongylus spp. and Nematodirus spp. (Fig. 2), while Trichuris spp. were $100 \%$ susceptible on all three farms. Trichuris spp. were susceptible to all three drugs, except on farm number 3, where levamisole did not have this $100 \%$ effectiveness. The larval culture and FECR results revealed that $\mathrm{AR}$ was present in the Arbat District, primarily as a characteristic of Trichostrongylus and, to a lesser extent, Nematodirus species.

Larval development assay. The LDA was used to confirm the FECRT results and determine the in vitro $\mathrm{IC}_{50}$ of albendazole, ivermectin, and levamisole. The results are shown in Table 2. The LDA results revealed that $0.3 \mu \mathrm{g} / \mathrm{mL}$ of albendazole completely inhibited nematode larval development in vitro on all three farms. The median inhibitory concentrations for albendazole on the three farms were $0.076 \pm 0.004 \mu \mathrm{g} / \mathrm{mL}$, $0.071 \pm 0.004 \mu \mathrm{g} / \mathrm{mL}$, and $0.072 \pm 0.007 \mu \mathrm{g} / \mathrm{mL}$, respectively. This outcome indicated that albendazole effectively inhibited larval nematode development at the recommended concentrations.

Table 1. Percentage of third-stage larvae in the first 100 larvae isolated from a pooled post-treatment faecal sample of the control and treated groups

\begin{tabular}{ccccc}
\hline \multirow{2}{*}{ Farm } & Pre-treatment FEC $($ eggs/g) & \multicolumn{3}{c}{ Percentage of larvae by genus } \\
\cline { 2 - 5 } & $764.1 \pm 45.2$ & Trichostrongylus & Nematodirus & Trichuris \\
\hline 1 & $760.0 \pm 48.1$ & 82.0 & 12.0 & 6.0 \\
\hline 2 & $604.2 \pm 37.8$ & 76.0 & 15.0 & 9.0 \\
\hline 3 & 86.0 & 8.0 & 6.0 \\
\hline
\end{tabular}

* Values represent mean pre-treatment FECs in 40 sheep \pm SEM

Table 2. Median inhibitory concentration $\left(\mathrm{IC}_{50}\right)$ of anthelmintics preventive of nematode egg hatchability

\begin{tabular}{cccc}
\hline \multirow{2}{*}{ Farm } & & $\mathrm{IC}_{50}$ & Levamisole $(\mu \mathrm{g} / \mathrm{mL})$ \\
\cline { 2 - 4 } & Albendazole $(\mu \mathrm{g} / \mathrm{mL})$ & Ivermectin $(\mathrm{ng} / \mathrm{mL})$ & $1.470 \pm 0.090$ \\
\hline 1 & $0.076 \pm 0.004$ & $8.030 \pm 0.692$ & $1.450 \pm 0.140$ \\
3 & $0.071 \pm 0.004$ & $7.670 \pm 0.622$ & $1.390 \pm 0.120$ \\
\hline
\end{tabular}

Values represent the mean of three tests \pm SEM 


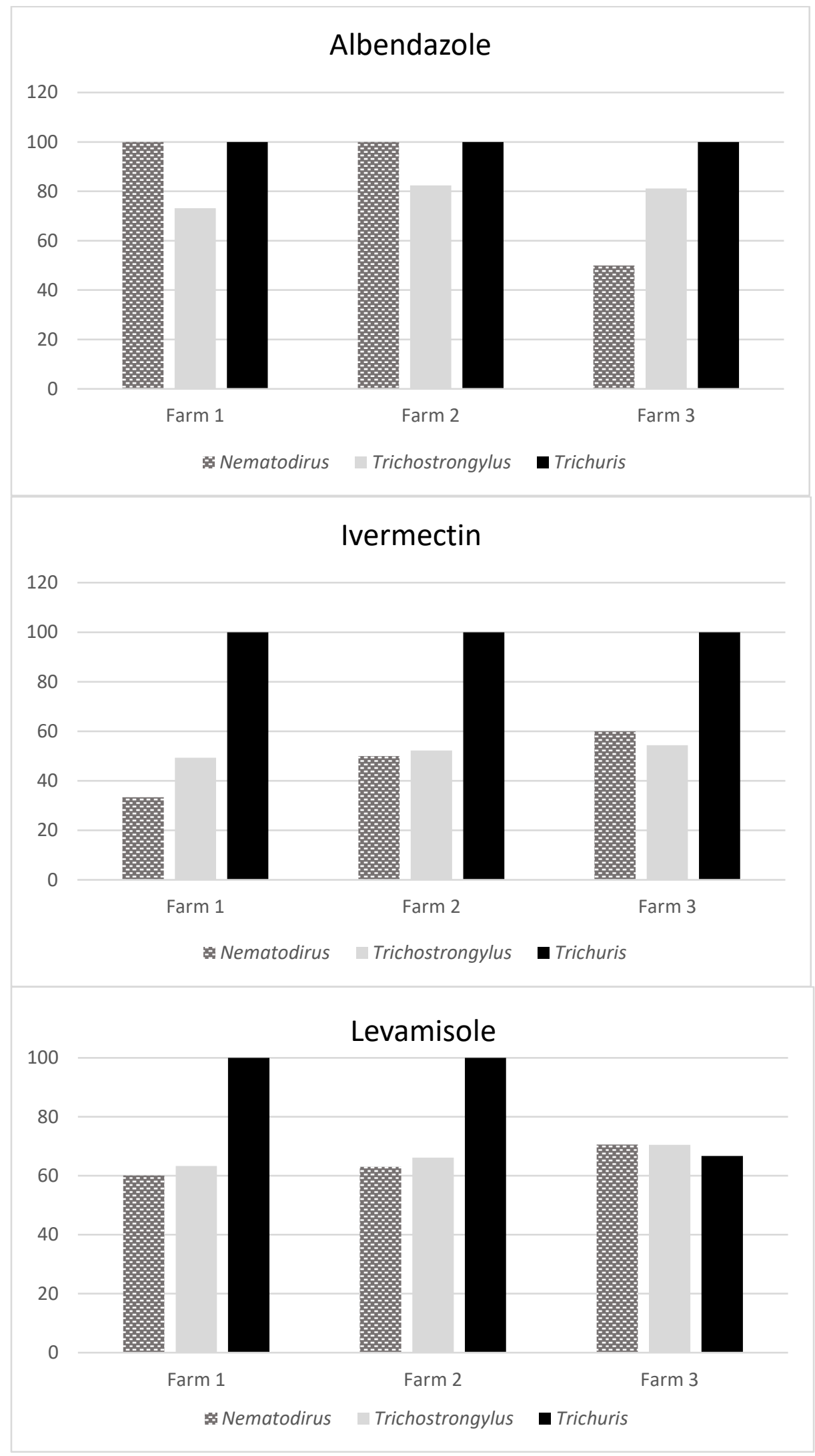

Fig. 2. Percentage of reduction in Nematodirus spp., Trichostrongylus spp., and Trichuris spp. after treatment with anthelmintics on Arbat's three sheep farms 
Levamisole was also effective in inhibiting larval development, as a $3.0 \mu \mathrm{g} / \mathrm{mL}$ concentration inhibited approximately $82 \%$ of the larvae in all three farms. The $\mathrm{IC}_{50}$ of levamisole were $1.47 \pm 0.09 \mu \mathrm{g} / \mathrm{mL}$, $1.45 \pm 0.14 \mu \mathrm{g} / \mathrm{mL}$, and $1.39 \pm 0.12 \mu \mathrm{g} / \mathrm{mL}$ for the three farms. These results showed that the nematode larvae were susceptible to levamisole's inhibitory effects at the standard concentrations.

Ivermectin did not effectively inhibit larval developments at the recommended concentration of $3.5 \mathrm{ng} / \mathrm{mL}$, and only at a concentration as high as $31.5 \mathrm{ng} / \mathrm{mL}$ did it inhibit larvae, doing so for about $79 \%$. Ivermectin's $\mathrm{IC}_{50}$ values were $8.03 \pm 0.69 \mathrm{ng} / \mathrm{mL}$, $7.67 \pm 0.62 \mathrm{ng} / \mathrm{mL}$, and $8.20 \pm 0.62 \mathrm{ng} / \mathrm{mL}$, respectively.

The LDA results showed that albendazole was the most effective anthelmintic drug at inhibiting nematode larval development at the prescribed concentration. Levamisole inhibited larval progress to L3 at concentrations slightly higher than the recommendations. Ivermectin, in contrast, was ineffective in inhibiting larvae at the recommended concentration, and the $\mathrm{IC}_{50}$ was about 2.3 times as high as that value.

\section{Discussion}

Anthelmintic resistance is an increasing problem of small ruminant production worldwide that may render currently available drugs inefficient in the future, and accordingly, the development of control strategies has become a necessity (13). Implementing a suitable AR control program starts with identifying the common GINs and determining the extent of resistance in an area (15). In the north of Iraq, where the livestock industry is the key agricultural sector, GIN control is highly important, and investigating how AR is distributed among GIN species is required to improve the control strategy.

In the study area, GIN control is primarily dependent on anthelmintic treatment. This study investigated AR in Arbat District after reports of anthelmintic failure on several farms in the area. FECRT, considered the most reliable method of testing an anthelmintic drug's utility under field conditions (18), was used to elucidate the efficacy of albendazole, ivermectin, and levamisole in vivo. However, the method cannot identify the specific GINs in a given study area, and therefore larval cultures were conducted concomitantly to determine the GINs parasitising Arbat District sheep. The in vitro LDA was also used to confirm the FECRT results. The findings of this study confirmed the occurrence of AR to all the tested drugs, as none of them was able to reduce FECs by $95 \%$. The larval cultures revealed that Trichostrongylus spp., Nematodirus spp., and Trichuris spp. were the common GINs in the study area. Also, it was shown that the reported resistance is due to Trichostrongylus and to a lesser extent Nematodirus spp.
The FECRT showed that ivermectin was the least efficacious anthelmintic in this study, while albendazole was the most effective. The questionnaire answers revealed that the drugs most commonly used by farmers in the study area were ivermectin, levamisole and albendazole, in order of frequency of implementation. Frequent use of an anthelmintic over an extended period usually provokes AR development (21). Ivermectin is an endectocide often used to control external arthropods and internal nematode parasites, which may justify its frequent use by sheep farmers. The drug's frequent use might be the reason why it was the least effective anthelmintic.

One of the FECRT disadvantages is that the results' accuracy is affected by such factors as underdosing. The tested drugs were administered to each animal to overcome this problem using recommended dosage rates based on each sheep's weight. Therefore, the AR reported by the FECRT cannot be a result of deficient dose administration and must only be due to the emergence of resistant GINs. Furthermore, the FECRT results were confirmed using the in vitro LDA.

The LDA was conducted to determine the drugs' $\mathrm{IC}_{50}$ values, and the results indicated that $\mathrm{AR}$ against ivermectin had developed on all three farms. The drug's $\mathrm{IC}_{50}$ ranged between 7.67 and $8.20 \mathrm{ng} / \mathrm{mL}$, while previous studies reported $\mathrm{IC}_{50}$ of $0.23 \mathrm{ng} / \mathrm{mL}(16,20)$, meaning that ivermectin was only $3 \%$ as potent in the present study. In a previous study, the IC50 of levamisole on Trichuris muris larvae was $1.75 \mu \mathrm{g} / \mathrm{mL}$ (27), close to the $1.39 \mu \mathrm{g} / \mathrm{mL}$ reported in this study. This result opposed the in vivo FECRT, as AR to levamisole was evident in the latter. Albendazole showed a potent effect in the LDA, as the drug's $\mathrm{IC}_{50}$ of $0.071-0.076$ $\mu \mathrm{g} / \mathrm{mL}$ was in the range of recommended concentrations (10). This outcome also contrasted with the FECRT results. Many factors may lead to a false finding of decreased efficacy, such as defective equipment, wrong dosage, missed animals, inferior product, and mislabelled samples (17). However, these factors might not cause the discrepancy between these LDA results and those of the FECRT, as care was taken to avoid misconduct in executing the procedures. The daily egg production differs in different nematode species, affecting anthelmintic efficacy during FECRT (24), and because the effect of this variable was not eliminated in the study, it may explain why the LDA and FECRT results are at variance.

In conclusion, this study is the first report which reveals AR occurrence in sheep in Arbat District, northern Iraq, confirmed by FECRT and LDA. Resistance to ivermectin was evident and was principally due to the emergence of resistant Trichostrongylus species. The common GIN genera in the study area were Trichostrongylus, Nematodirus, and Trichuris. The results establish the ground for designing and executing an appropriate GIN control program in Arbat and other areas in the north of Iraq. 
Conflict of Interest Statement: The authors declare that there is no conflict of interests regarding the publication of this article.

Financial Disclosure Statement: The University of Sulaimani provided the funds to conduct this study.

Animal Rights Statement: All procedures that include animals were approved by the Animal Care and Use Committee at the University of Sulaimani under approval number AUP-2020-09.

\section{References}

1. Abongwa M., Martin R.J., Robertson A.P.: A brief review on the mode of action of antinematodal drugs. Acta Vet 2017, 67, 137-152, doi: 10.1515/acve-2017-0013.

2. Bosco A., Kießler J., Amadesi A., Varady M., Hinney B., Ianniello D., Maurelli M.P., Cringoli G., Rinaldi L.: The threat of reduced efficacy of anthelmintics against gastrointestinal nematodes in sheep from an area considered anthelmintic resistance-free. Parasit Vectors 2020, 13, 1-12, doi: 10.1186/s13071-020-04329-2.

3. Claerebout E., De Wilde N., Van Mael E., Casaert S., Velde F.V., Roeber F., Veloz P.V., Levecke B., Geldhof P.: Anthelmintic resistance and common worm control practices in sheep farms in Flanders, Belgium. Vet Parasitol Reg Stud Reports 2020, 20, 100393, doi: 10.1016/j.vprsr.2020.100393.

4. Coles G., Bauer C., Borgsteede F., Geerts S., Klei T., Taylor M., Waller P.: World Association for the Advancement of Veterinary Parasitology (WAAVP) methods for the detection of anthelmintic resistance in nematodes of veterinary importance. Vet Parasitol 1992, 44, 35-44, doi: 10.1016/0304-4017(92)90141-U.

5. Coles G., Jackson F., Pomroy W., Prichard R., von SamsonHimmelstjerna G., Silvestre A., Taylor M., Vercruysse J.: The detection of anthelmintic resistance in nematodes of veterinary importance. Vet Parasitol 2006, 136, 167-185, doi: 10.1016/j.vetpar.2005.11.019

6. Colgrave M.L., Kotze A.C., Kopp S., McCarthy J.S., Coleman G.T., Craik D.J.: Anthelmintic activity of cyclotides: in vitro studies with canine and human hookworms. Acta Trop 2009, 109, 163-166, doi: 10.1016/j.actatropica.2008.11.003.

7. Craig T.M.: Gastrointestinal nematodes, diagnosis and control. Vet Clin North Am Small Anim Pract 2018, 34, 185-199, doi: 10.1016/j.cvfa.2017.10.008.

8. Dyary H.O.: Veterinary anthelmintics and anthelmintic drug resistance. J Zankoy Sulaimani A 2016, 18, 191-206, doi: 10.17656/jzs.10463.

9. Dyary H.O.: Anthelmintic resistance of gastrointestinal nematodes in sheep in Piramagroon sub-district, Sulaymaniyah/ Iraq. Trop Biomed 2018, 35, 373-382.

10. Falzon L., Menzies P., Shakya K., Jones-Bitton A., Vanleeuwen J., Avula J., Stewart H., Jansen J., Taylor M., Learmount J.: Anthelmintic resistance in sheep flocks in Ontario, Canada. Vet Parasitol 2013, 193, 150-162, doi: 10.1016/j.vetpar.2012.11.014.

11. French K.E.: Plant-based solutions to global livestock anthelmintic resistance. Ethnobiol Lett 2018, 9, 110-123, doi: 10.14237/ebl.9.2.2018.980

12. Giovanelli F., Mattellini M., Fichi G., Flamini G., Perrucci S.: In vitro anthelmintic activity of four plant-derived compounds against sheep gastrointestinal nematodes. Vet Sci 2018, 5, 78, doi: 10.3390/vetsci5030078.

13. Hodgkinson J.E., Kaplan R.M., Kenyon F., Morgan E.R., Park A.W., Paterson S., Babayan S.A., Beesley N.J., Britton C., Chaudhry U.: Refugia and anthelmintic resistance: concepts and challenges. Int J Parasitol Drugs Drug Resist 2019, 10, 51-57, doi: 10.1016/j.jpddr.2019.05.001.

14. Hubert J., Kerboeuf D.: A microlarval development assay for the detection of anthelmintic resistance in sheep nematodes. Vet Rec 1992, 130, 442-446, doi: 10.1136/vr.130.20.442.

15. Kaplan R.M.: Biology, epidemiology, diagnosis, and management of anthelmintic resistance in gastrointestinal nematodes of livestock. Vet Clin North Am Small Anim Pract 2020, 36, 17-30, doi: 10.1016/j.cvfa.2019.12.001.

16. Kotze A.C., Ruffell A.P., Knox M.R., Kelly G.A.: Relative potency of macrocyclic lactones in in vitro assays with larvae of susceptible and drug-resistant Australian isolates of Haemonchus contortus and H. placei. Vet Parasitol 2014, 203, 294-302, doi: 10.1016/j.vetpar.2014.04.005.

17. Levecke B., Dobson R., Speybroeck N., Vercruysse J., Charlier J.: Novel insights in the faecal egg count reduction test for monitoring drug efficacy against gastrointestinal nematodes of veterinary importance. Vet Parasitol 2012, 188, 391-396, doi: 10.1016/j.vetpar.2012.03.020.

18. Levecke B., Kaplan R.M., Thamsborg S.M., Torgerson P.R., Vercruysse J., Dobson R.J.: How to improve the standardisation and the diagnostic performance of the fecal egg count reduction test? Vet Parasitol 2018, 253, 71-78, doi: 10.1016/ j.vetpar.2018.02.004.

19. Mavrot F., Hertzberg H., Torgerson P.: Effect of gastrointestinal nematode infection on sheep performance: A systematic review and meta-analysis. Parasit Vectors 2015, 8, 557, doi: 10.1186/s13071-015-1164-z.

20. Raza A., Lamb J., Chambers M., Hunt P.W., Kotze A.C.: Larval development assays reveal the presence of sub-populations showing high-and low-level resistance in a monepantel (Zolvix $($ ))-resistant isolate of Haemonchus contortus. Vet Parasitol 2016, 220, 77-82, doi: 10.1016/j.vetpar.2016.02.031.

21. Sallé G., Cortet J., Bois I., Dubès C., Guyot-Sionest Q., Larrieu C., Landrin V., Majorel G., Wittreck S., Woringer E.: Risk factor analysis of equine strongyle resistance to anthelmintics. Int J Parasitol Drugs Drug Resist 2017, 7, 407-415, doi: 10.1016/j.ijpddr.2017.10.007.

22. Sangster N.C., Cowling A., Woodgate R.G.: Ten events that defined anthelmintic resistance research. Trends Parasitol 2018, 34, 553-563, doi: 10.1016/j.pt.2018.05.001.

23. Sorobetea D., Svensson-Frej M., Grencis R.: Immunity to gastrointestinal nematode infections. Mucosal Immunol 2018, 11, 304-315, doi: 10.1038/mi.2017.113.

24. Taylor M., Hunt K., Goodyear K.: Anthelmintic resistance detection methods. Vet Parasitol 2002, 103, 183-194, doi: 10.1016/S0304-4017(01)00604-5.

25. Tinkler S.H.: Preventive chemotherapy and anthelmintic resistance of soil-transmitted helminths - Can we learn nothing from veterinary medicine? One Health 2020, 9, 100106, doi: 10.1016/j.onehlt.2019.100106.

26. Van Wyk J.A., Mayhew E.: Morphological identification of parasitic nematode infective larvae of small ruminants and cattle: A practical lab guide. Onderstepoort J Vet Res 2013, 80, 539, doi: 10.4102/ojvr.v80i1.539.

27. Wimmersberger D., Tritten L., Keiser J.: Development of an in vitro drug sensitivity assay for Trichuris muris first-stage larvae. Parasit Vectors 2013, 6, 1-8, doi: 10.1186/1756-3305-642. 